


\title{
CAPTURE CROSS-SECTION MEASUREMENTS OF SOME MEDIUM- AND HEAVY-WEIGHT NUCLEI IN THE keV REGION ${ }^{\dagger}$
}

\author{
D. KOMPE
}

Institut fiir Angewandte Kernphysik, Kernforschungszentrum Karlsruhe, Karlsruhe, Germany

\author{
Received 17 February 1969
}

Abstract: The radiative neutron capture cross sections of $\mathrm{Nb}, \mathrm{Mo}, \mathrm{Pd}, \mathrm{Ag}, \mathrm{Cd}, \mathrm{In}, \mathrm{Cs}, \mathrm{Hf}, \mathrm{Ta}, \mathrm{W}$ and $\mathrm{Re}$ were measured relative to that of $\mathrm{Au}$ in the energy range form 10 to $150 \mathrm{keV}$ by the time-of-flight method. A large liquid scintillator was used as detector. The capture cross section of $\mathrm{Au}$ was determined from measurements performed relative to the ${ }^{10} \mathrm{~B}(\mathrm{n}, \alpha)^{7} \mathrm{Li}^{*}$ and ${ }^{6} \mathrm{Li}(\mathrm{n}, \alpha)^{3} \mathrm{H}$ reactions and relative to a flat-response neutron monitor; these three measurements were normalized at $30 \mathrm{keV}$. The data were analysed for $\mathrm{p}$ - and $\mathrm{d}$-wave strength functions.

NUCLEAR REACTIONS Nb, Mo, Pd, Ag, Cd, In, Cs, Hf, Ta, W, Re, Au(n, $\gamma$ ), $E=10-150 \mathrm{keV}$; measured $\sigma(E)$; deduced strength functions $S_{1}, S_{2}$.

\section{Introduction}

The knowledge of neutron radiative capture cross sections in the keV energy region is of interest for the design of fast reactors, for nuclear reaction theories such as the optical model, and for astrophysical theories.

In the past many cross sections for the capture of neutrons in the $\mathrm{keV}$ region have been investigated. Much effort has been spent to overcome the various difficulties of measurements in this energy region. Experimental and evaluation techniques have been improved, and in many cases the discrepancies are smaller now. But there is still a need for further measurements and improved accuracy.

Several methods for the measurement of capture cross sections in the $\mathrm{keV}$ region are in use nowadays. One of the earliest methods is the activation technique which allows the determination of absolute reaction rates in many cases with high accuracy. Its disadvantage is the limitation to radioactive final nuclei with convenient halflives.

The shell transmission method yields absolute capture cross sections at a few energies where monoenergetic neutron sources are available ${ }^{1,2}$ ). For the evaluation which is complicated by large multiple scattering and self-shielding corrections the application of Monte Carlo techniques ${ }^{3,4}$ ) was a great improvement.

The most generally applicable method of detecting a neutron capture event by the emitted prompt $\gamma$-radiation is utilized in the slowing-down-time spectrometer ${ }^{5,6}$ ) and

$\dagger$ Work performed within the association in the field of fast reactors between the European Atomic Energy Community and Gesellschaft für Kernforschung m.b.H., Karlsruhe. 
most frequently in the time-of-flight technique. Various neutron sources such as mechanical choppers, pulsed reactors, nuclear explosions, and pulsed accelerators are used.

The detectors for such experiments should fulfill several requirements: Good time resolution, insensitivity to inscattered neutrons and an efficiency independent of the form of the $\gamma$-ray cascade which may change from resonance to resonance in an unknown way. The last condition would be met ideally by a $4 \pi$-detector with $100 \%$ efficiency. Such a detector has been approximated in several laboratories by large liquid scintillator tanks ${ }^{7-9}$ ) up to 40001 [ref. $^{10}$ )].

A less bulky detector invented by Moxon and Rae ${ }^{11}$ ) achieves independence from the $\gamma$-ray cascade mode by its proportionality between efficiency and $\gamma$-ray energy. This detector has been successfully used with linear accelerators, but its small efficiency makes it less useful at a Van de Graaff. A collimator for $\mathrm{keV}$ neutrons would require a flight path of the order of $0.5 \mathrm{~m}$, but the neutron intensities from a Van de Graaff are very low at such distances. On the other hand, application of kinematic collimation near the threshold of the neutron-producing reaction limits the energy range, if one requires that the detector is not hit by the neutron cone. Moreover the energy resolution for this detector is worse than that obtainable with a large liquid scintillator where nearly the same time resolution can be achieved at a flight path which is an order of magnitude longer.

Recently some improvements in efficiency over the original Moxon-Rae detector were published. A many-layer-detector was developed by Weigmann et al. ${ }^{12}$ ). But the efficiency of $2.4 \cdot 10^{-3} / \mathrm{MeV}$ is still rather small for Van de Graaff applications.

Maier-Leibnitz proposed another detector with much higher efficiency which was developed by Macklin et al. ${ }^{13}$ ). Contrary to the Moxon-Rae version this detector has an efficiency depending nonlinearly upon energy. Therefore a more complicated two-dimensional analysis of pulse-height versus time-of-flight spectra and a correction for the nonlinear efficiency is necessary.

For these reasons we chose a large liquid scintillation detector for our time-of-flight experiments at the $3 \mathrm{MV}$ pulsed Van de Graaff accelerator at Karlsruhe. With this detector we measured the neutron capture cross sections for the elements $\mathrm{Nb}, \mathrm{Mo}$, $\mathrm{Pd}, \mathrm{Ag}, \mathrm{Cd}, \mathrm{In}, \mathrm{Cs}, \mathrm{Hf}, \mathrm{Ta}, \mathrm{W}$ and $\mathrm{Re}$ relative to the capture cross section of $\mathrm{Au}$ in the energy range from 10 to $160 \mathrm{keV}$. The capture cross section of gold used as standard in the present work was obtained from three independent measurements of the cross section shape which were then normalized to an absolute value at $30 \mathrm{keV}$.

$\mathrm{p}$ - and d-wave strength functions were determined from an analysis of the experimental curves which takes into account the first three partial waves and inelastic scattering.

\section{Apparatus and techniques}

\subsection{THE LIQUID SCINTILLATION TANK}

Our large liquid scintillation detector is shown schematically in fig. 1. The aluminium container has cylindrical symmetry about the vertical axis and approximates 
a sphere. It has a diameter of $1.1 \mathrm{~m}$ and a volume of 8001 . The sample is placed inside a central aluminium tube, $10 \mathrm{~cm}$ in diameter and $1 \mathrm{~mm}$ thick. Absorption of $\mathrm{MeV}$ $\gamma$-rays in the tube walls is negligible. The inner surfaces of the tank are coated with a

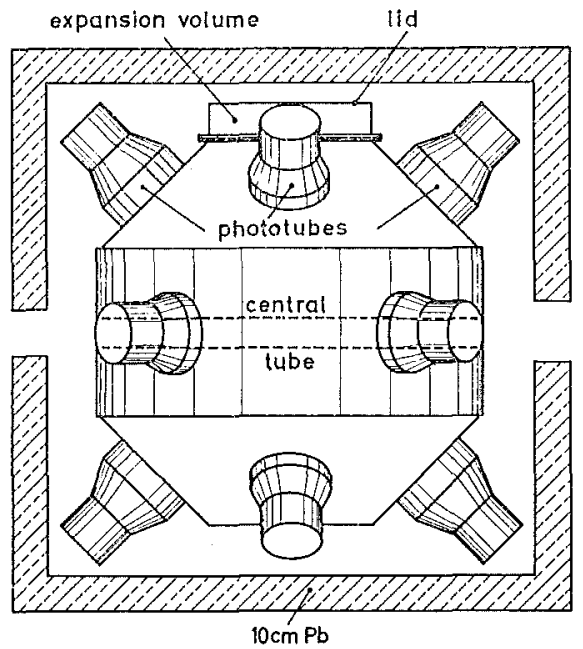

Fig. 1. The scintillation tank.

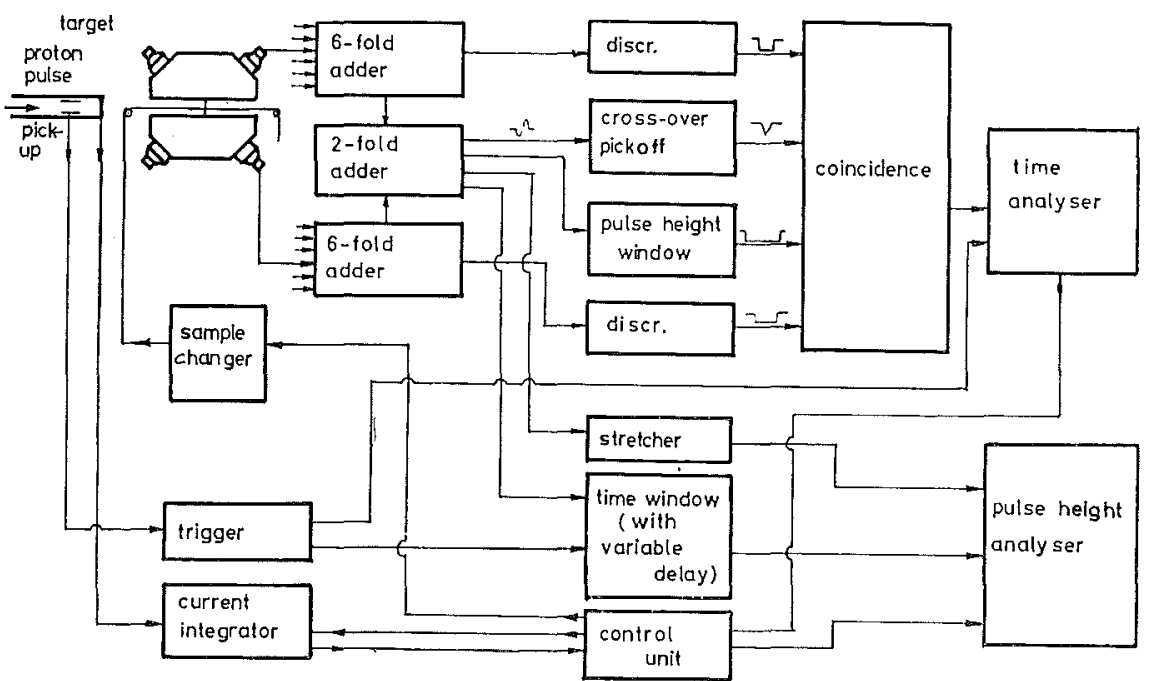

Fig. 2. Block diagram of the electronic apparatus for the scintillation tank.

commercially available reflector paint, an epoxy resin containing titanium dioxide (NE 561).

The liquid scintillator is a solution of p.terphenil $(5 \mathrm{~g} / 1)$ and POPOP $(0.1 \mathrm{~g} / 1)$ in pseudocumene $\left(1,2,4\right.$-trimethylbenzene). This solvent has a high flash point $\left(54^{\circ} \mathrm{C}\right)$ 
and gives a good light output ${ }^{14}$ ). About $1 \%$ trimethylborate was added to suppress the $2.2 \mathrm{MeV} \gamma$-ray background from the capture of inscattered neutrons by the hydrogen of the scintillator.

The scintillator is viewed by 12 Valvo $57-A V P$ photomultipliers, $20 \mathrm{~cm}$ in diameter, which are in direct contact with the liquid. A Viton O-ring seals the glass of each phototube to its container; the container is sealed by an indium wire to the tank. Fig. 2 shows a block diagram of the electronics. The gain of each phototube can be adjusted by a potentiometer on a high voltage fan-out. Emitter followers driven by the high voltage source act as impedance transformers to the cables which are adjusted in length to achieve good overall time resolution. The single signals are fed to two 6-fold adder circuits. A coincidence between these two groups suppresses noise pulses. The sum signal from the two branches delivers the timing and pulse-height information of an event. For timing a fast tunnel diode crossover pickoff was used. The time resolution measured with a $1 \mathrm{nsec} \gamma$-ray flash produced by the proton beam from the accelerator at the center of the tank was 3 nsec. Time-of-flight spectra were taken within a pulse-height window which was chosen from 3 to $12 \mathrm{MeV}$ to get a good signal-tobackground ratio. Simultaneously with the time-of-flight spectra pulse-height spectra down to $1 \mathrm{MeV} \gamma$-ray energy are measured within a time window set on the neutron peak; background is determined by shifting the time window off the neutron peak. Width and mutual delay of the time windows are very stable since they are given by cable length. An automatic sample changer transports the sample suspended from two steel wires into the center of the tank. During the relative measurements the two samples were interchanged from different sides of the tank at short time intervals (about 5 minutes) controlled by a current integrator for the proton beam. Thus the influence of time dependent variations on the relative counting rates is eliminated.

The efficiency of the detector for $\gamma$-rays originating at the centre of the tank may be written as the product

$$
\varepsilon=p_{\mathrm{i}} p_{\mathrm{b}}
$$

where $p_{\mathrm{i}}$ is the probability for at least one interaction with the scintillator and $p_{\mathrm{b}}$ is the probability that the resulting detector signal exceeds the discriminator bias level.

The interaction probability $p_{i 1}$ for monoenergetic $\gamma$-rays of energy $E$ can easily be calculated by an integration over the tank volume:

$$
p_{\mathrm{i} 1}(E)=1-\overline{\mathrm{e}^{-\mu(E) R}},
$$

where $R$ is the scintillator thickness in the direction of emission; the absorption coefficients $\mu(E)$ were taken from ref. ${ }^{49}$ ). The resulting $p_{\mathrm{i} 1}$ for our scintillation tank is given by the curve $n=1$ in fig. 3 . The detector is nearly black only for small $\gamma$ energies. The value $1-p_{\mathbf{i} 1}(E=0)=0.004$ corresponds to $\gamma$-ray escape through the central hole. The probability $p_{\text {in }}$ that at last one $\gamma$-quantum out of a cascade with the multiplicity $n$ and total energy $E=\sum_{v=1}^{n} E_{v}$ interacts with the scintillator can be 
calculated from (2) by

$$
p_{\mathrm{in}}(E)=1-\prod_{\nu=1}^{n}\left[1-p_{\mathrm{i} 1}\left(E_{v}\right)\right]
$$

In practice, this formula is not yet very useful, since the complicated $\gamma$-ray spectra after neutron capture and their possible dependences on neutron energy are not known.

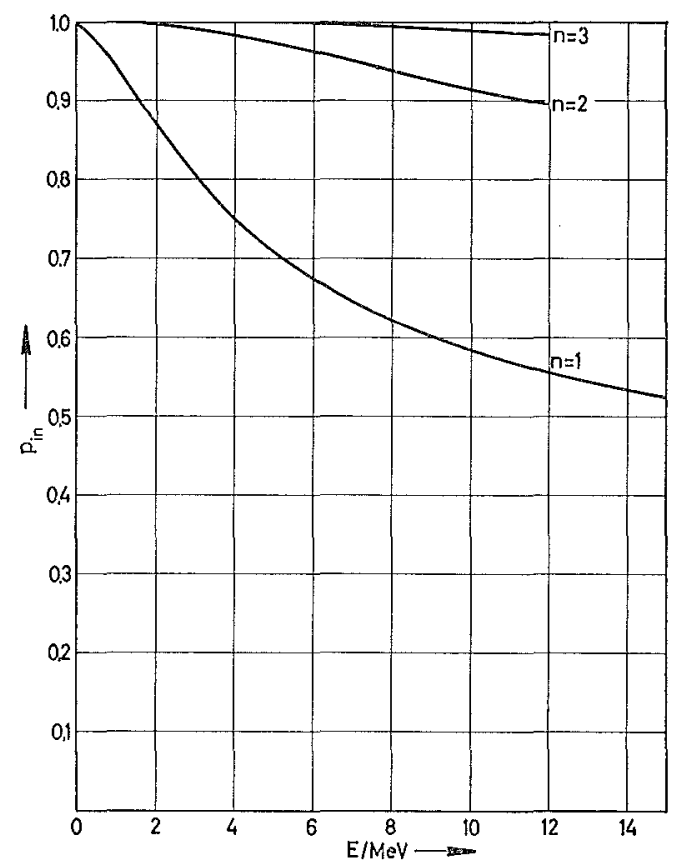

Fig. 3. Probability $p_{\text {in }}$ for at least one interaction of a $\gamma$-ray cascade of multiplicity $n$ with the scintillator.

However, a good estimate can be found from the case where all $E_{v}$ are equal. For a given $n$ and $E=n E_{v}, p_{\text {in }}(E)$ then takes its lower limit because of the curvature of $p_{\mathrm{i} 1}(E)$. This most unfavourable case

$$
p_{\mathbf{i} n}(E)=1-\left[1-p_{i 1}\left(\frac{E}{n}\right)\right]^{n},
$$

is shown for $n=2,3$ in fig. 2. Since the average multiplicity of $\gamma$-ray cascades for the heavy nuclei investigated here is usually greater than 3 the interaction probability is obviously close to 1 . We assume that the estimated value

$$
p_{\text {in }}=0.97 \pm 0.03 \text {, }
$$

should encompass even unfavourable cases with some admixture of high-energy transitions. 
For the determination of the fraction $p_{\mathrm{b}}$ of pulses above the discriminator bias level an analysis of the pulse-height spectrum is necessary. For $(n, \gamma)$ reactions this procedure will be discussed later.

The energy resolution of the detector was tested with several $\boldsymbol{\gamma}$-ray sources. Measured values for 3 different energies are given in table 1 .

TABLE 1

Resolution of the liquid scintillation tank

\begin{tabular}{ccc}
\hline$\gamma$-source & $\begin{array}{c}\gamma \text {-ray energy } \\
\text { (total) }\end{array}$ & Energy resolution \\
\hline${ }^{137} \mathrm{Cs}$ & $0.661 \mathrm{MeV}$ & $49 \%$ \\
${ }^{60} \mathrm{Co}$ & $2.5 \mathrm{MeV}$ & $25 \%$ \\
${ }^{24} \mathrm{Na}$ & $4.12 \mathrm{MeV}$ & $20 \%$ \\
\hline
\end{tabular}

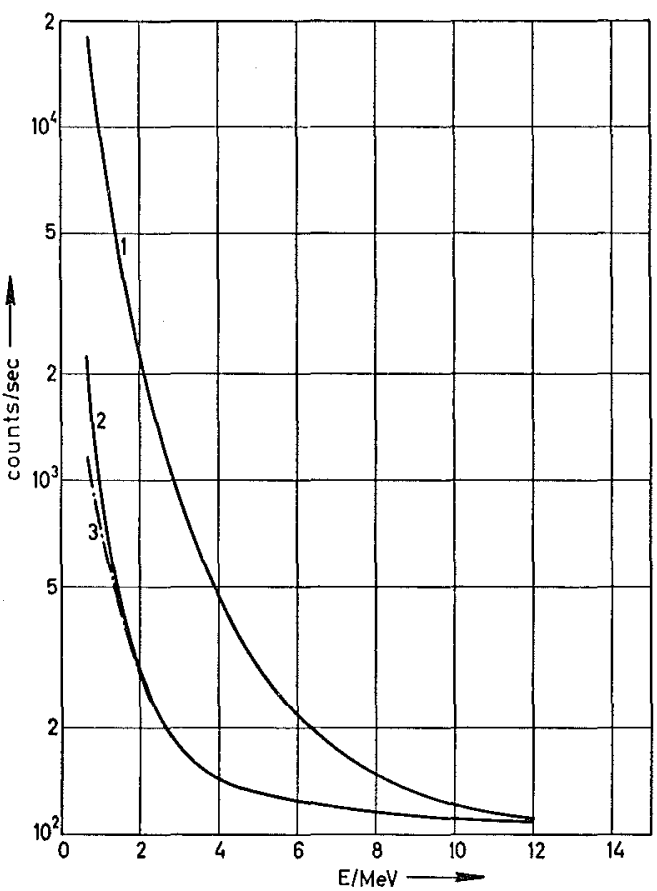

Fig. 4. Background counting rates above discriminator bias at energy $E$ without shielding (1), with $10 \mathrm{~cm}$ of lead shielding (2), and with lead shielding and coincidence (3).

The detector is shielded on all sides by $10 \mathrm{~cm}$ of lead. Fig. 4 shows the integrated background counting rates as a function of discriminator bias without (curve 1) and with (curve 2) lead shielding. The coincidence arrangement between the two groups of phototubes for noise suppression gives only a small reduction in background counting rates at low energies, as is shown by curve 3 . 


\subsection{EXPERIMENTAL PROCEDURE}

The capture cross section measurements were performed at the $3 \mathrm{MV}$ Van de Graaff accelerator which delivered an average proton current of $2-4 \mu \mathrm{A}$ at $10 \mathrm{nsec}$ pulse width and $0.5 \mathrm{MHz}$ repetition frequency. A broad neutron spectrum from 10 to $150 \mathrm{keV}$ was produced by the ${ }^{7} \mathrm{Li}(\mathrm{p}, \mathrm{n})^{7} \mathrm{Be}$ reaction in a thick $\mathrm{Li}$ metal target. The target is placed in the center of a Li-paraffin collimator of $1.2 \mathrm{~m}$ side length which reduces the neutron-induced background. The well-collimated neutron beam hits the sample inside the tank after a flight path of $1.5 \mathrm{~m}$. The timing resolution was determined by the proton pulse width which was large compared to the timing resolution of the detector; the resulting resolution of the spectrometer was $7 \mathrm{~ns} / \mathrm{m}$. That corresponds to an energy resolution of $2 \%$ at $10 \mathrm{keV}$ and $7.5 \%$ at $150 \mathrm{keV}$.

The sample diameter of $9 \mathrm{~cm}$ exceeds the neutron beam diameter so that a small vertical displacement of the sample has no influence. Metallic samples of about $1 \mathrm{~mm}$ thickness were used. The $\mathrm{Cs}$ sample consisted of $\mathrm{CsCl}$ powder packed in a thin-walled container. The small capture cross section of $\mathrm{Cl}$ can be neglected; however, its scattering cross section has to be taken into account for the multiple scattering correction.

For a comparison of the shape of the capture cross section of gold with that of the ${ }^{6} \mathrm{Li}(\mathrm{n}, \alpha)^{3} \mathrm{H}$ cross section a $\mathrm{Li}$ glass detector was used. A $3 \mathrm{~mm}$ thick ${ }^{6} \mathrm{Li}$ glass scintillator within a $0.1 \mathrm{~mm}$ thick bronze container was placed normal to the photocathode of an XP 1040 photomultiplier. A thin aluminium foil reflector on the inside of the container gives sufficient light collection and good energy resolution for the neutroninduced peak. When placed behind the liquid scintillator tank this detector introduces only a minimum of scattering material into the collimated neutron beam.

In a similar way a boron slab detector described by $\mathrm{Rohr}^{15}$ ) was used for the measurement relative to the shape of the ${ }^{10} \mathrm{~B}(\mathrm{n}, \alpha)^{7} \mathrm{Li}^{*}$ cross section. In this case a thin slab of ${ }^{10} \mathrm{~B}$ powder $\left(2.5 \cdot 10^{22}\right.$ atoms $\left./ \mathrm{cm}^{2}\right)$ was viewed by four surrounding $\mathrm{NaI}$ crystals.

The boron slab detector which has a good signal-to-background ratio was used to verify that the collimator does not produce a time-dependent background of scattered neutrons. In a time-of-flight test measurement with monoenergetic neutrons from a thin target no time dependent neutron background was found within an accuracy of $2 \%$.

\subsection{EVALUATION METHODS}

The capture cross section $\sigma_{\gamma}$ of a sample with $N$ atoms per unit area measured relative to a standard cross section $\sigma_{\mathrm{s}}$ is

$$
\sigma_{\gamma}(E)=\frac{R(E) N_{\mathrm{s}} C_{\mathrm{s}}(E) \varepsilon_{\mathrm{s}}}{R_{\mathrm{s}}(E) N C(E) \varepsilon} \sigma_{\mathrm{s}},
$$

where $R, R_{\mathrm{s}}$ are the detector counting rates for the two samples after background subtraction. $C$ and $C_{\mathrm{s}}$ are correction factors for multiple scattering and resonance selfshielding varying between 0.99 and 1.08 for our samples. They were calculated from approximations that Macklin ${ }^{16}$ ) has derived from Dresner's formulas ${ }^{17}$ ). 
For the smooth ${ }^{10} \mathrm{~B}$ cross section the scattering correction of Schmitt ${ }^{18}$ ) was used. In the case of Li-glass the correction factor was interpolated from values that Zetterström et al. have calculated by the Monte Carlo method ${ }^{19}$ ).

Since $p_{\text {in }}$ is assumed constant (see eq. (5)), $p_{\mathrm{bs}} / p_{\mathrm{b}}$, the ratio of the spectrum fiactions above the discriminator bias used for the time-of-flight spectra, can be substituted for $\varepsilon_{\mathrm{s}} / \varepsilon$, the ratio of the detector efficiencies for the two samples. The pulse-height spectra necessary for the determination of these spectrum fractions were measured simultaneously with the time-of-flight spectra. The high background which was determined by shifting the time gate off the neutron peak does not allow a measurement of the pulse-height spectra below $\gamma$-ray energies of $1 \mathrm{MeV}$. Therefore one must extrapolate to zero pulse height and this procedure introduces an estimated uncertainty of $5 \%$. Such an extrapolation is reasonable since all processes resulting in a partial loss of the total $\gamma$-ray energy are continuous, and hence the low-energy part of the capture pulseheight spectrum is a smooth curve. The low detector resolution has an additional smoothing effect on the pulse-height spectra.

The extrapolation method is further justified by the similarity of the different capture pulse-height spectra. Assuming a uniform shape for all capture pulse-height spectra and adjusting the energy scale to the neutron binding energy the spectrum fractions $p_{\mathrm{b}}$ were calculated for the different elements. The difference between calculated and measured spectrum fractions was only $8 \%$. This indicates that the influence of the form of the $\gamma$-ray spectrum on the pulse-height spectrum is not very strong. The estimated uncertainty in $p_{b}$ of $5 \%$ does not seem to be too optimistic.

The statistical error in the background-corrected counting rates $R, R_{\mathrm{s}}$ becomes rather large below $20 \mathrm{keV}$. Therefore the statistical accuracy between 10 and $20 \mathrm{keV}$ was improved by combining channels in the time-of-flight spectra; as a result the energy resolution is reduced.

For the measurements of the gold cross section the flight path to the reference detector behind the tank was $0.9 \mathrm{~m}$ longer than that to the gold sample inside the tank. Since the transmission of an air path of that length increases $2 \%$ over our energy range, a correction for this effect had to be applied.

\section{Results and discussion}

\subsection{THE CAPTURE CROSS SECTION OF GOLD}

The capture cross section of gold in the $\mathrm{keV}$ region has been frequently investigated. Although some earlier measurements showed discrepancies up to a factor of two, there is now good agreement between several independent measurements with good accuracy around $30 \mathrm{keV}$. A detailed discussion of this situation is given in ${ }^{20,21}$ ). We have previously $\left.{ }^{21}\right)$ adopted $\sigma_{\mathrm{Au}(\mathrm{n}, \gamma)}(30 \mathrm{keV})=0.596 \pm 0.012 \mathrm{~b}$ as an average value from the activation and shell transmission measurements and measurements relative to ${ }^{10} \mathrm{~B}(\mathrm{n}, \alpha)$ of several groups. This value was then used for the normalization of the gold cross section measurement reported in that paper. In that experiment, performed with 
monoenergetic neutrons from a thin target at several energies, neutron capture in the gold sample was detected by the large liquid scintillator. The neutron flux was monitored by a flat-response counter ("grey neutron detector") ${ }^{22}$ ). An accuracy of about $5 \%$ was obtained for that measurement.

For the present investigation the gold cross section is needed with the resolution observed in the thick-target time-of-flight measurements. Therefore two additional time-of-flight measurements relative to the ${ }^{10} \mathrm{~B}(\mathrm{n}, \alpha)^{7} \mathrm{Li}$ and ${ }^{6} \mathrm{Li}(\mathrm{n}, \alpha)^{3} \mathrm{H}$ reaction cross sections were performed using the detectors described in subsect. 2.2. Both measurements were normalized to the $30 \mathrm{keV}$ value given above.

The ${ }^{10} \mathrm{~B}(\mathrm{n}, \alpha \gamma)$ cross section was based on a $1 / v$ energy dependence of the ${ }^{10} \mathrm{~B}(\mathrm{n}, \alpha)$ cross section and the branching ratio $b=1-\sigma_{\alpha \gamma} / \sigma_{\alpha}$ that Spaepen has recommended [ref. ${ }^{23}$ )]. Spaepen assumes a $1 / v$ behaviour for $\sigma_{\alpha}$ up to $100 \mathrm{keV}$, but the experimental results he evaluated are compatible with $1 / 0$ within a $5 \%$ uncertainty up to at least $150 \mathrm{keV}$. This was confirmed by Cox et al. ${ }^{24}$ ) who found recently a $1 / \mathrm{v}$ dependence up to $250 \mathrm{keV}$. The branching ratio $b$ is also uncertain by about $5 \%$. However its contribution to the error in $\sigma_{\alpha \gamma}=(1-b) \sigma_{\alpha}$ is small compared to the $5 \%$ uncertainty in $\sigma_{\alpha}$, since in our energy range $b<0.1$.

The experimental data for the ${ }^{6} \mathrm{Li}(\mathrm{n}, \alpha)^{3} \mathrm{H}$ cross section in our energy range agree only to about $15 \%$ in their absolute values, but rather well in shape. As reference cross section we used the fitted Breit-Wigner curve that Schwartz et al. ${ }^{25}$ ) obtained from their measured points. Considering the data from the different experiments that Schwartz has compared we estimate that this cross section shape is correct to about $5 \%$.

For the two measurements of the gold cross section relative to ${ }^{10} \mathrm{~B}$ and ${ }^{6} \mathrm{Li}$ we get the following errors:

(i) Uncertainty in the shape of the reference cross section: $5 \%$.

(ii) Normalization error at $30 \mathrm{keV}: 3 \%$.

(iii) Uncertainties in multiple scattering corrections: $3 \%$.

(iv) Uncertainty in the correction for air scattering within the flight path difference between the two detectors: $0.5 \%$.

(v) Possible variation of the spectrum fraction above bias with neutron energy: $4 \%$.

(vi) Statistical error: $3.5 \%$ at $10 \mathrm{keV}$, less than $1 \%$ above $30 \mathrm{keV}$.

The resulting total error for these two measurements decreases from $8.5 \%$ at $10 \mathrm{keV}$ to $3 \%$ at the normalization point $30 \mathrm{keV}$, and rises again to $8 \%$ at higher energies.

The results of these two measurements which agree very well are shown in fig. 5 . Fig. 6 shows an average of these two curves and the result of the measurement using the grey neutron detector where lower resolution was obtained. There is again good agreement at low energies, whereas a slight deviation occurs above $80 \mathrm{keV}$. The standard cross section assumed for the present work is also shown in fig. 6. It represents a weighted average of all three measurements with the resolution of the two time-offlight measurements relative to ${ }^{10} \mathrm{~B}$ and ${ }^{6} \mathrm{Li}$. The estimated error is indicated for a few points. 
At the high-energy end this cross section standard is $15 \%$ below a previously published preliminary result ${ }^{20,26}$ ) which was based on a measurement relative to a $\mathrm{Li}$ glass detector. That detector had been placed at the same location as the gold sample inside the tank; however, the large scattering mass in the neutron beam caused a large time-dependent background that was incorrectly subtracted.

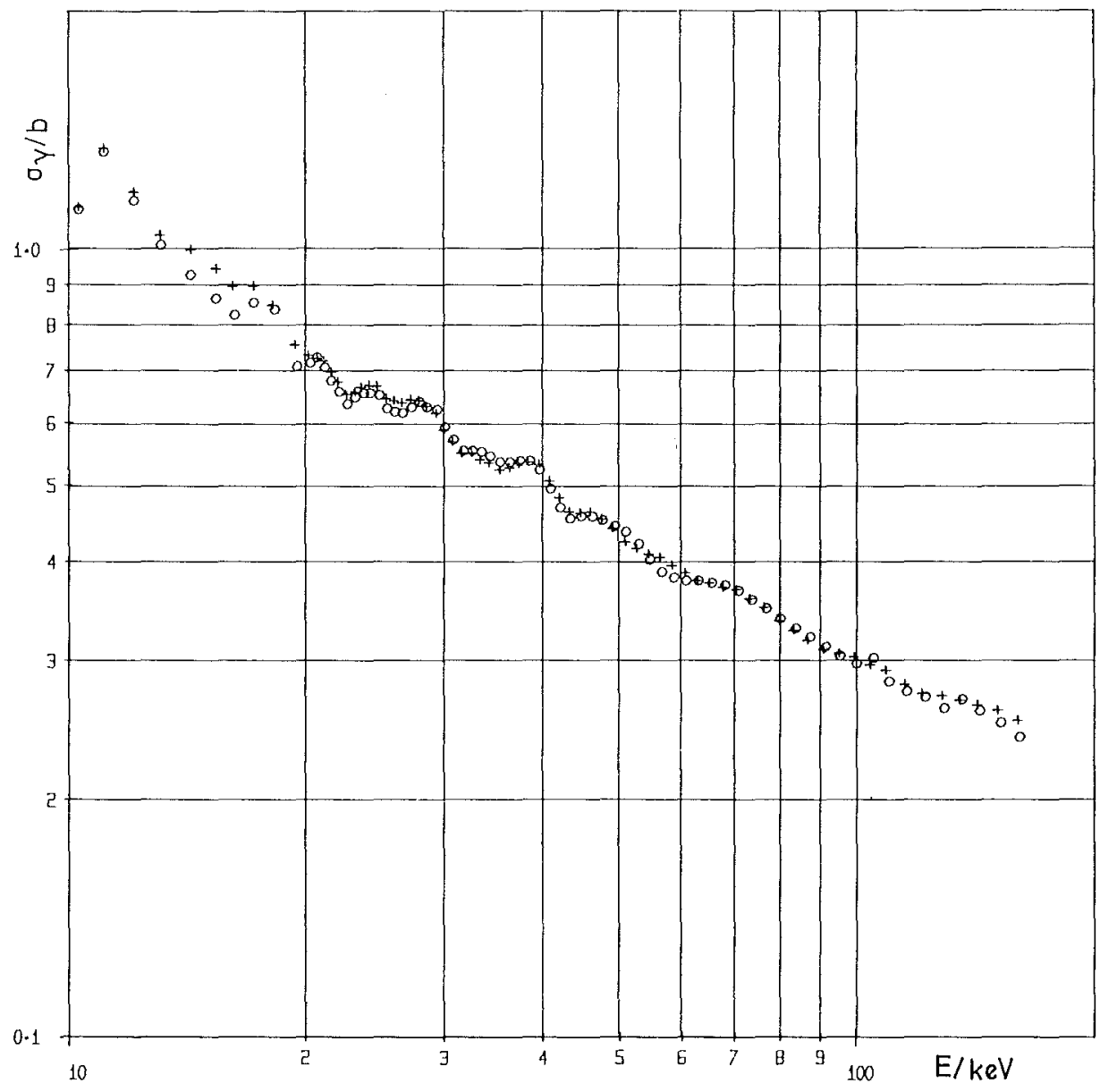

Fig. 5. The capture cross section of Au measured relative to ${ }^{10} \mathrm{~B}(+++)$ and ${ }^{6} \mathrm{Li}(\mathrm{O} O \mathrm{O})$.

The gold cross section was already discussed in detail in ${ }^{21}$ ). We had found good agreement with various other measurements, especially in the lower-energy region. In the region above $130 \mathrm{keV}$ our results are about $15 \%$ lower than those based on the fission cross section of ${ }^{235} \mathrm{U}$ as measured by White ${ }^{27}$ ). The reason of this discrepancy is not clear. 


\subsection{CROSS SECTIONS MEASURED RELATIVE TO GOLD}

For the error in the capture cross sections measured relative to the standard cross section of gold we get the following contributions from the quantities in eq. (6):

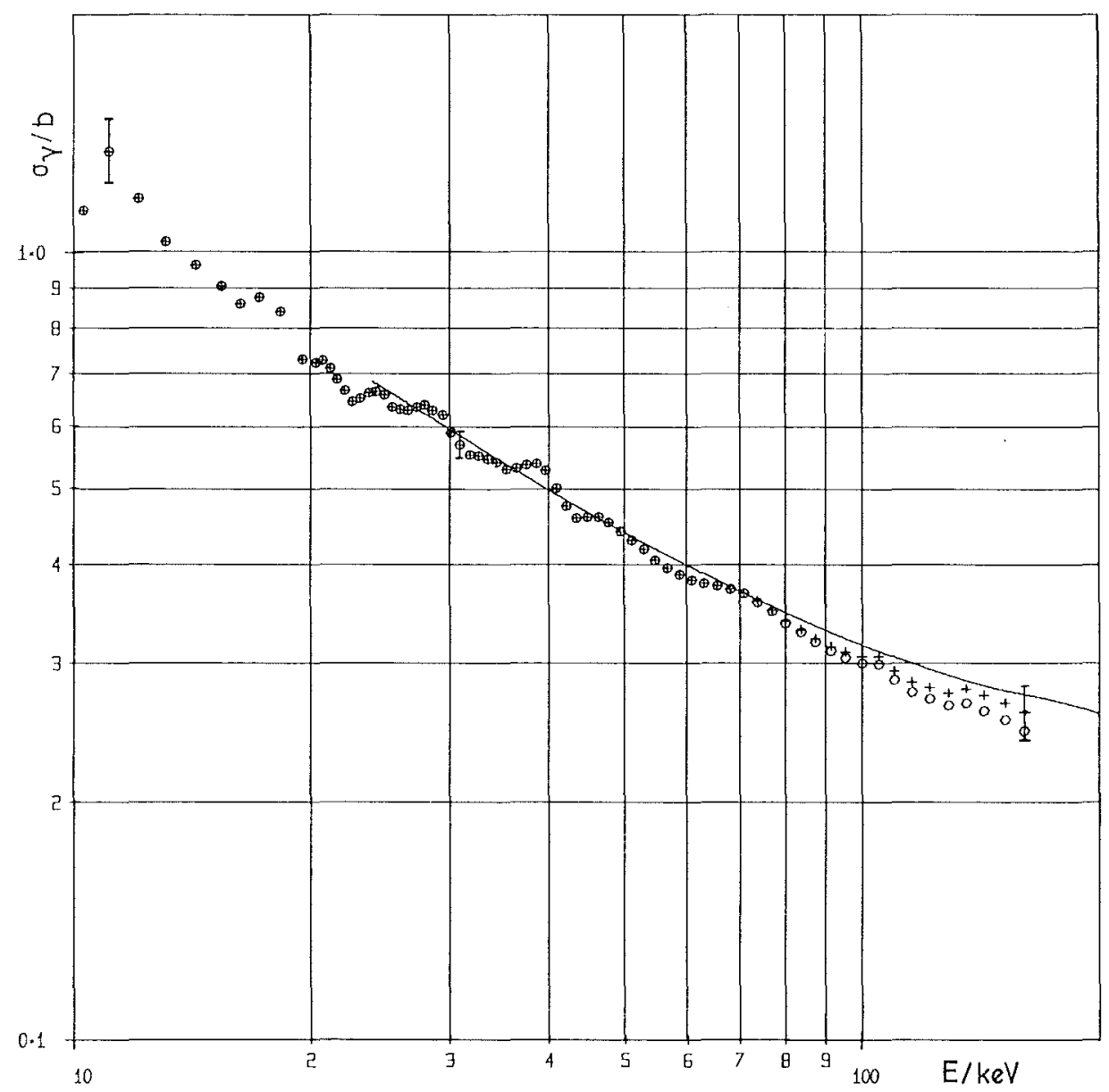

Fig. 6. The standard gold cross section $(+++)$, resulting from the measurements relative to the grey neutron detector (-), and to ${ }^{10} \mathrm{~B}$ and ${ }^{6} \mathrm{Li}$ which are represented here by an average curve ( $0 \circ 0)$.

(i) Error in the standard cross section of gold $\sigma_{\mathrm{s}}: 3-8 \%$.

(ii) Uncertainty in the spectrum fraction above bias: $5 \%$.

(iii) Uncertainty in the interaction probability of $\gamma$-rays with the scintillator $p_{\mathrm{i}}: 3 \%$.

(iv) Error in the multiple scattering and self-shielding correction $C: 1 \%$.

(v) Statistical error in $R / R_{\mathrm{s}}: 8 \%$ at $10 \mathrm{keV}, 2.5 \%$ at $30 \mathrm{keV}$, and less than $1.5 \%$ above $50 \mathrm{keV}$. 
The resulting total error is then

at $10 \mathrm{keV} 13 \%$, at $20 \mathrm{keV} 11 \%$, at $30 \mathrm{keV} 8 \%$, above $50 \mathrm{keV} 10 \%$.

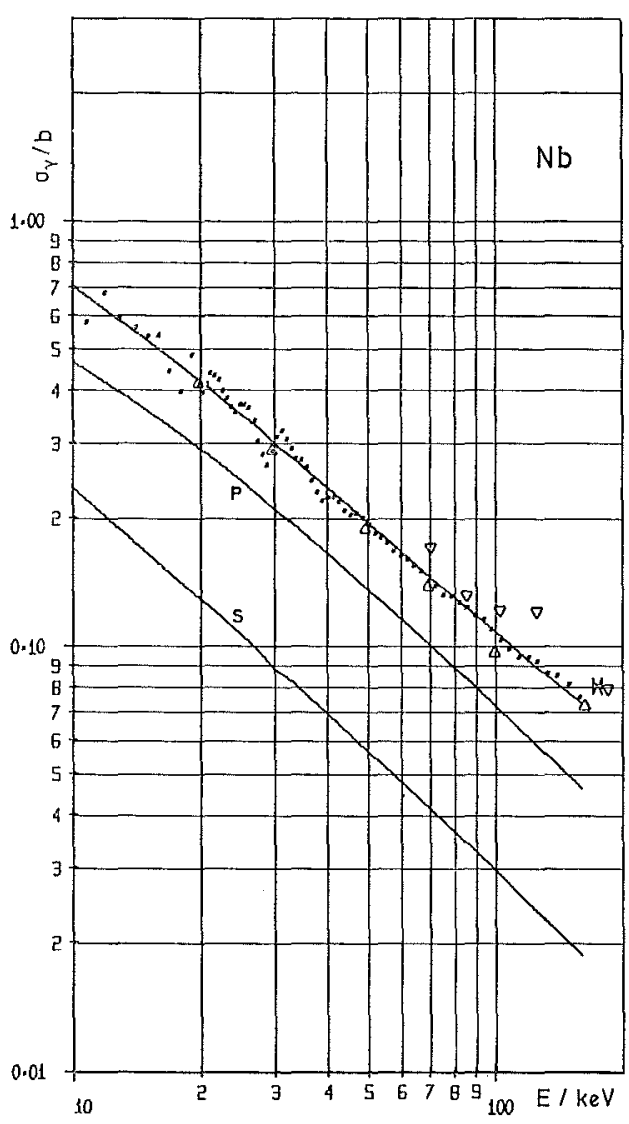

Fig. 7. Capture cross section of $\mathrm{Nb}$ $\triangle$ Gibbons et $a l .{ }^{8}$ ), $\nabla$ Macklin et al. ${ }^{13}$ ), $\circlearrowleft$ Diven et al. ${ }^{7}$ ).

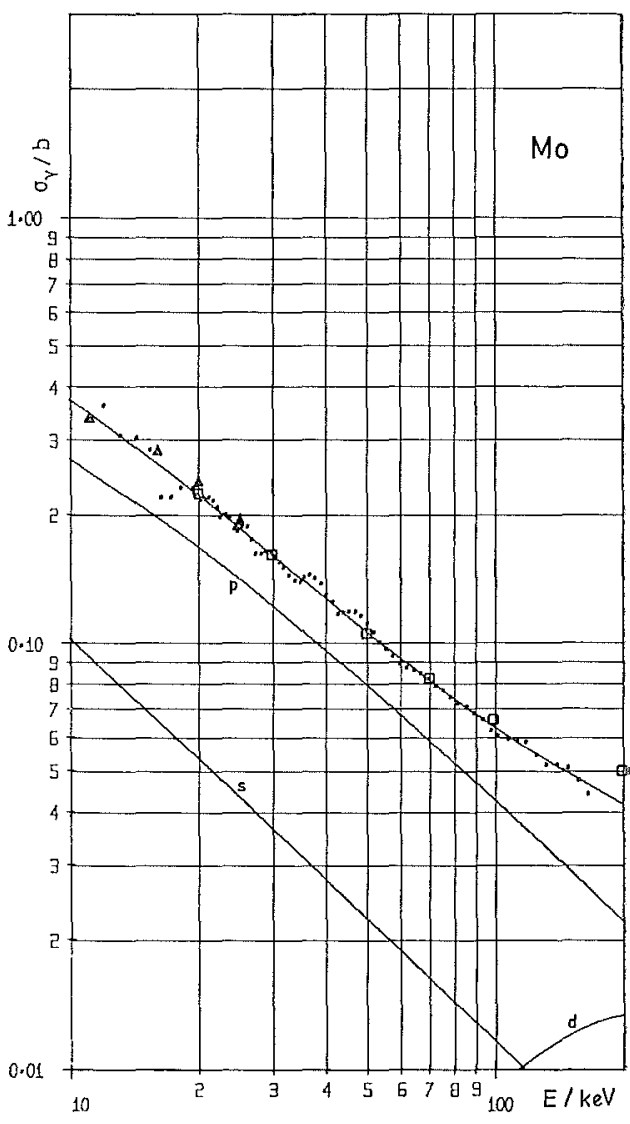

Fig. 8. Capture cross section of Mo $\square$ recommended by Poenitz ${ }^{20}$ ), A Weigmann et al. ${ }^{47}$ ).

In figs. 7-17 the results for the elements $\mathrm{Nb}, \mathrm{Mo}, \mathrm{Pd}, \mathrm{Ag}, \mathrm{Cd}, \mathrm{In}, \mathrm{Cs}, \mathrm{Hf}, \mathrm{Ta}, \mathrm{W}, \mathrm{Re}$ (represented by points) and fitted curves which will be discussed later are shown. They differ from the previously published preliminary results ${ }^{26}$ ) because of the new gold reference cross section as described above. For some of these elements Poenitz had recommended cross section curves ${ }^{20}$ ) which referred to the previous preliminary gold cross section. These recommended curves which are now renormalized to the new standard cross section and the results of other authors are also shown in the figures for comparison.

Considering the limits of error, the capture cross sections of this work for Mo, $\mathrm{Ag}$, In, and Ta (figs. 8, 10, 12, 15) agree quite well with the recommended values that 
Poenitz ${ }^{20}$ ) had extracted from other experiments. The agreement for $\mathrm{W}$ is not quite as good but it is within the limits of error. New measurements of Macklin and Gibbons ${ }^{13}$ ) for $\mathrm{Ag}$ and $\mathrm{W}$ agree well with our data. Unfortunately their gold cross section is about $15 \%$ lower so that the agreement in the corresponding cross section ratios is not so good. A recent measurement of Weigmann et al. ${ }^{47}$ ) for Mo below

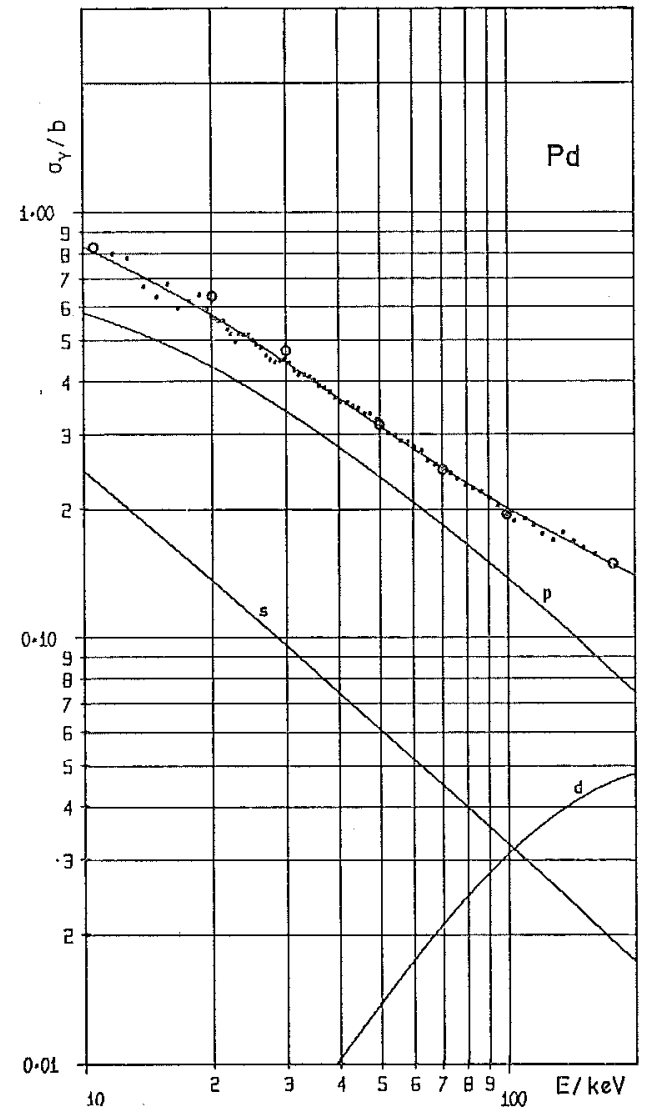

Fig. 9. Capture cross section of Pd: O BNL 325/ Suppl. 1.

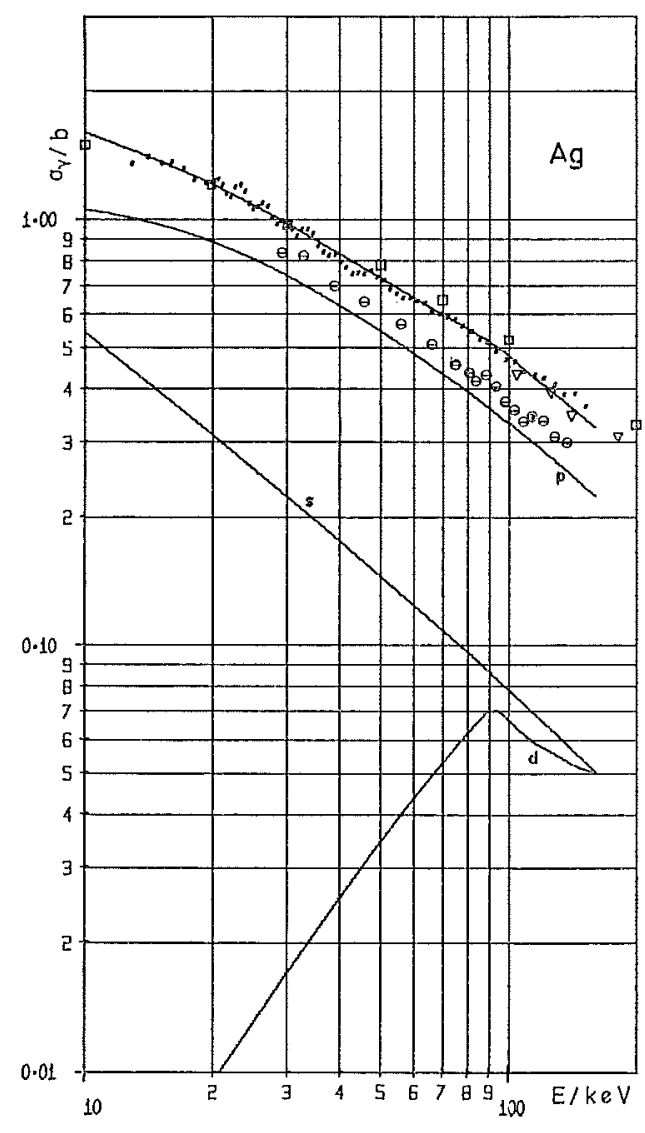

Fig. 10. Capture cross section of Ag: $\square$ recommended by Poenitz $\left.{ }^{20}\right), \nabla$ Macklin et al. ${ }^{13}$ ), $\ominus$ Kononov et $a l .{ }^{31}$ ).

$25 \mathrm{keV}$ joins our data very well. Their data are normalized at low energies by the "black resonance technique". The flux shape was measured versus the ${ }^{10} \mathrm{~B}(\mathrm{n}, \alpha)$ cross section. The good agreement with this absolute measurement is a further indirect confirmation of our gold cross section at low energies. The data of Kononov et al. ${ }^{31}$ ) for $\mathrm{Ag}, \mathrm{Ta}, \mathrm{W}$, and Re, which are normalized to the shell transmission measurements of Belanova et al. ${ }^{2}$ ), are systematically below our results by more than $20 \%$. 
For Nb, Pd, Cd (figs. 7, 9 and 11) some data from Oak Ridge as given in BNL 325 are shown for comparison. There is good agreement with the results of Gibbons et $a l .{ }^{8}$ ) within the limits of error whereas the new measurement of Macklin and Gibbons ${ }^{13}$ ) for $\mathrm{Nb}$ is about $10 \%$ above our curve. It should be mentioned again that the agreement would be worse in some cases after a renormalization to the same

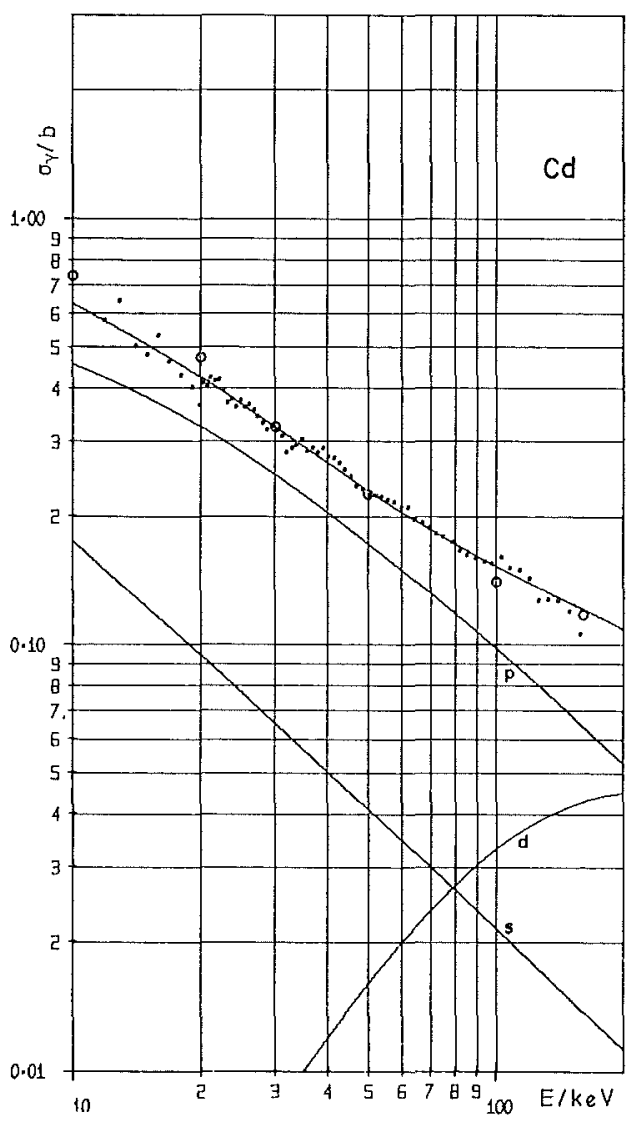

Fig. 11. Capture cross section of Cd: O BNL 325/ Suppl. 1.

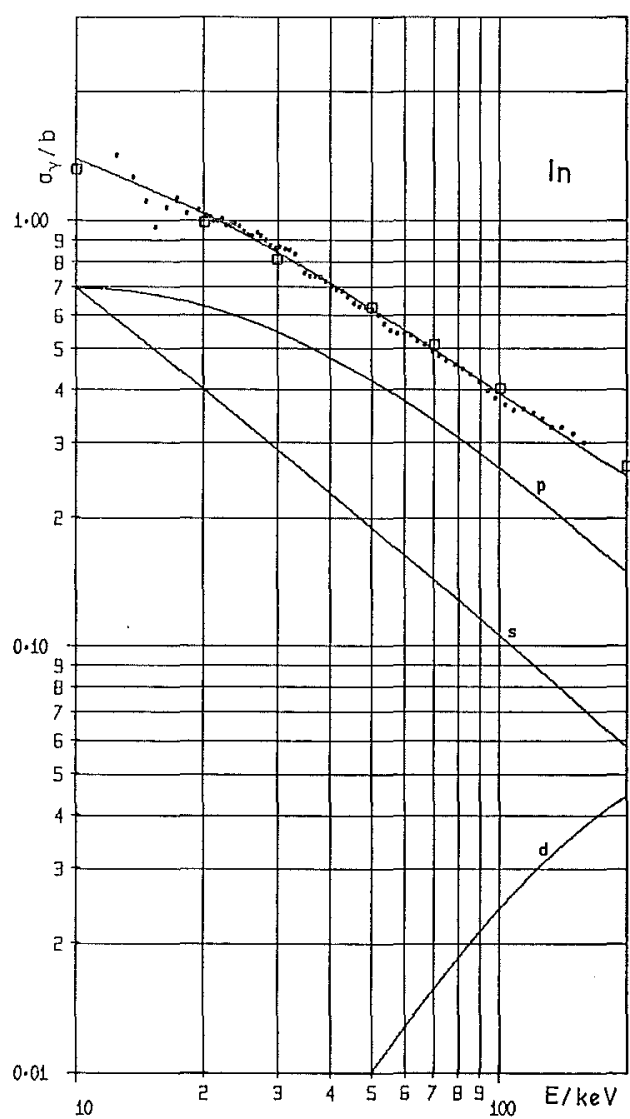

Fig. 12. Capture cross section of In: $\square$ recommended by Poenitz ${ }^{20}$ ).

gold cross section. There is one further point for $\mathrm{Nb}$ at $175 \mathrm{keV}$ from the measurement of Diven et al. ${ }^{7}$ ) which is about $15 \%$ above our data; but it should be noted that Diven obtained a $30 \%$ higher gold cross section.

For Cs (fig. 13), which is an important fission product in fast reactors, the lead spectrometer results of Popov and Shapiro ${ }^{28}$ ) below $45 \mathrm{keV}$ are about $30 \%$ below our curve. This deviation might be due to normalization difficulties at the slowingdown-time spectrometer. There is good agreement with an activation measurement at 24 
$\mathrm{keV}$ reported by Booth et al. ${ }^{29}$ ); however, the accuracy of this measurement was only $33 \%$.

For Hf and Re (figs. 14, 17) the measurements of Macklin et al. ${ }^{30}$ ) are appreciably below our data. The deviations which are about $30 \%$ for Hf and nearly $50 \%$ for Re may be only partly due to their lower normalization cross section. A measurement of

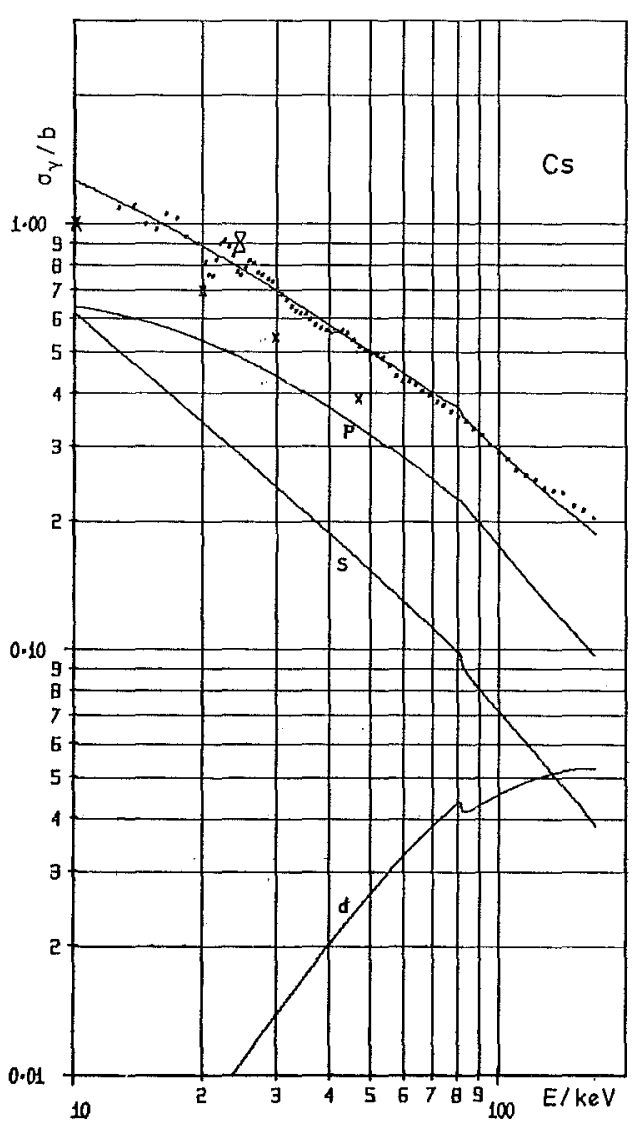

Fig. 13. Capture cross section of Cs: $\times$ Popov et al. ${ }^{28}$ ), $\square$ Booth et al. ${ }^{29}$ ).

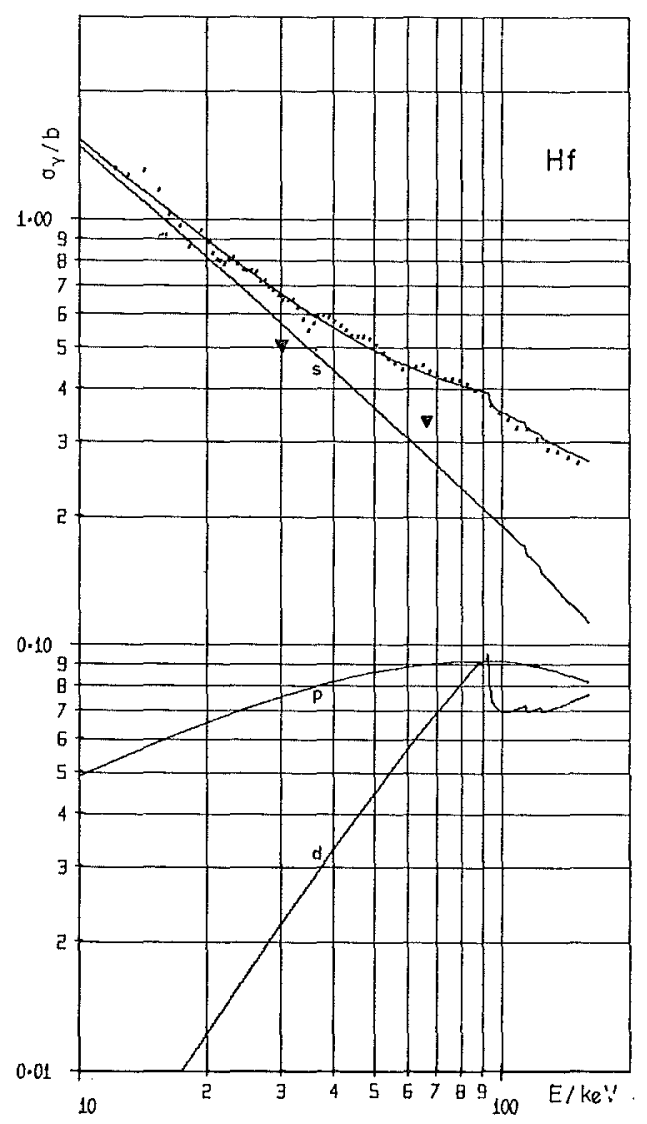

Fig. 14. Capture cross section of Hf: $\nabla$ Macklin et al. ${ }^{30}$ ).

Block et al. ${ }^{9}$ ) for $\mathrm{Hf}$ below $8 \mathrm{keV}$ seems to join our data quite well. The present data for Re are very well confirmed below $30 \mathrm{keV}$ by a recent absolute measurement of Friesenhahn et $a l .{ }^{32}$ ) which was normalized by the black resonance technique at low energies. The results of Stupegia et al. ${ }^{33}$ ) which are based on the fission cross section of ${ }^{235} \mathrm{U}$ as measured by White ${ }^{27}$ ) are about $20 \%$ above our curve, but they agree well in shape. Thus we again find the discrepancy with the fission cross section of ${ }^{235} \mathrm{U}$ which was already mentioned in the discussion of the gold cross section. 


\section{Analysis of average capture cross sections}

\subsection{THEORY}

From an analysis of our average capture cross sections we determined neutron strength functions and compared them to optical-model values. We shall therefore give a short survey of the theory that was used to describe our experimental results.

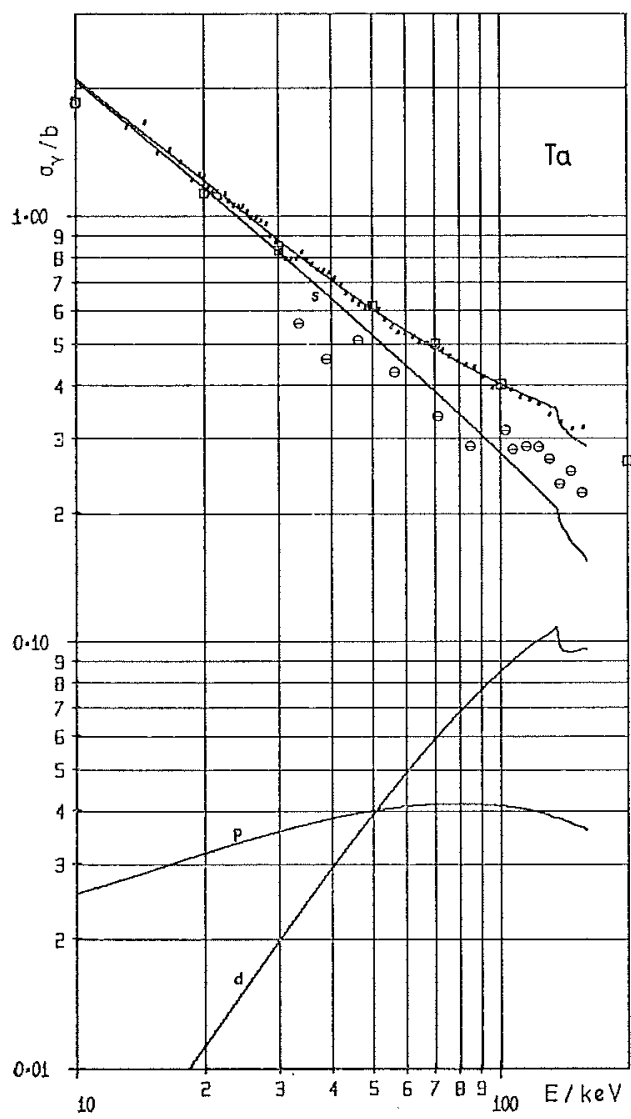

Fig. 15. Capture cross section of Ta: $\square$ recommended by Poenitz ${ }^{20}$ ), Kononov et al. ${ }^{31}$ ).

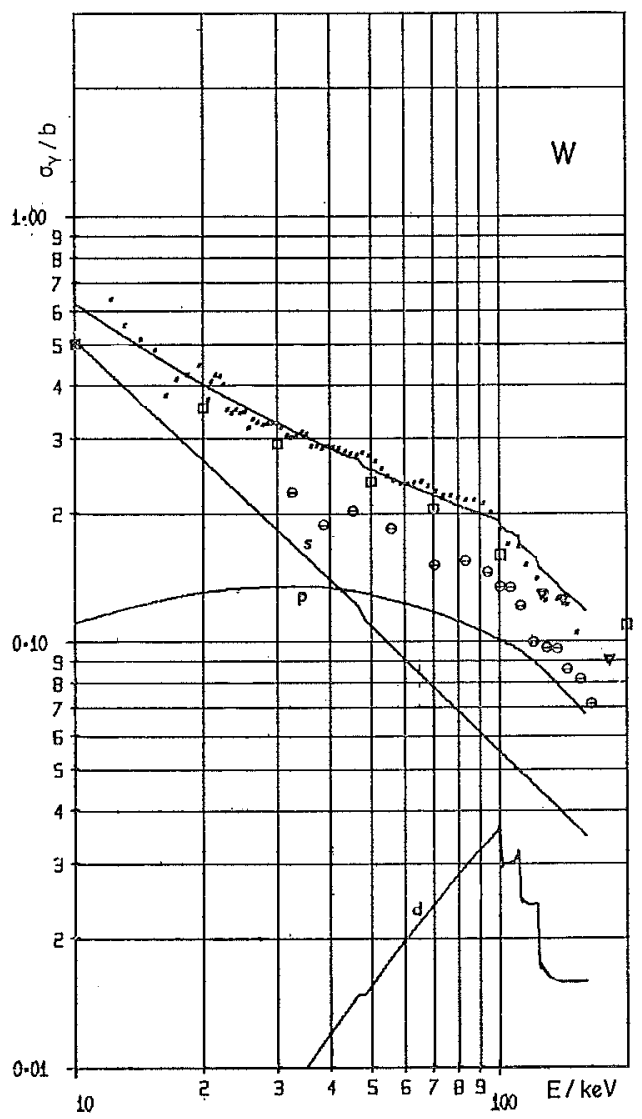

Fig, 16. Capture cross section of W: $\square$ recommended by Poenitz ${ }^{20}$ ), $\nabla$ Macklin et al. ${ }^{13}$ ), Kononov et al. ${ }^{31}$ ).

For heavy nuclei the experiment usually averages over many well-separated resonances. The average capture cross section for a total spin $J$ and an angular momentum $l$ of the neutron may then be written (see e.g. ref. ${ }^{34}$ ))

$$
\left\langle\sigma_{\gamma}^{J l}\right\rangle=2 \pi^{2} \hbar^{2} g_{J} \frac{1}{D_{J}} \frac{\left\langle\sum_{j} \Gamma_{\mathbf{n}}^{J l j}\right\rangle\left\langle\Gamma_{\gamma}^{J}\right\rangle}{\left\langle\Gamma^{J}\right\rangle} F,
$$


where $t$ is the reduced width of the neutron in the c.m. system, $g_{J}=(2 J+1) / 2(2 I+1)$, $I$ is the spin of the target nucleus, $D^{J}$ the average spacing of levels with spin $J,\left\langle\Gamma_{\gamma}^{J}\right\rangle$ the average radiation width, $\left\langle\Gamma_{\mathrm{n}}^{J l j}\right\rangle$ the average neutron width of the incoming neutron with channel spin $j=I \pm \frac{1}{2}$ for $I \neq 0$ and $j=\frac{1}{2}$ for $I=0$. The average total width is

$$
\left\langle\Gamma^{J}\right\rangle=\left\langle\Gamma_{\gamma}^{J}\right\rangle+\left\langle\sum_{l, j} \Gamma_{\mathrm{n}}^{J l j}\right\rangle+\left\langle\sum_{\mathrm{n}^{\prime}, l^{\prime}, j^{\prime}} \Gamma_{\mathrm{n}^{\prime}}^{J l^{\prime} j^{\prime}}\right\rangle
$$

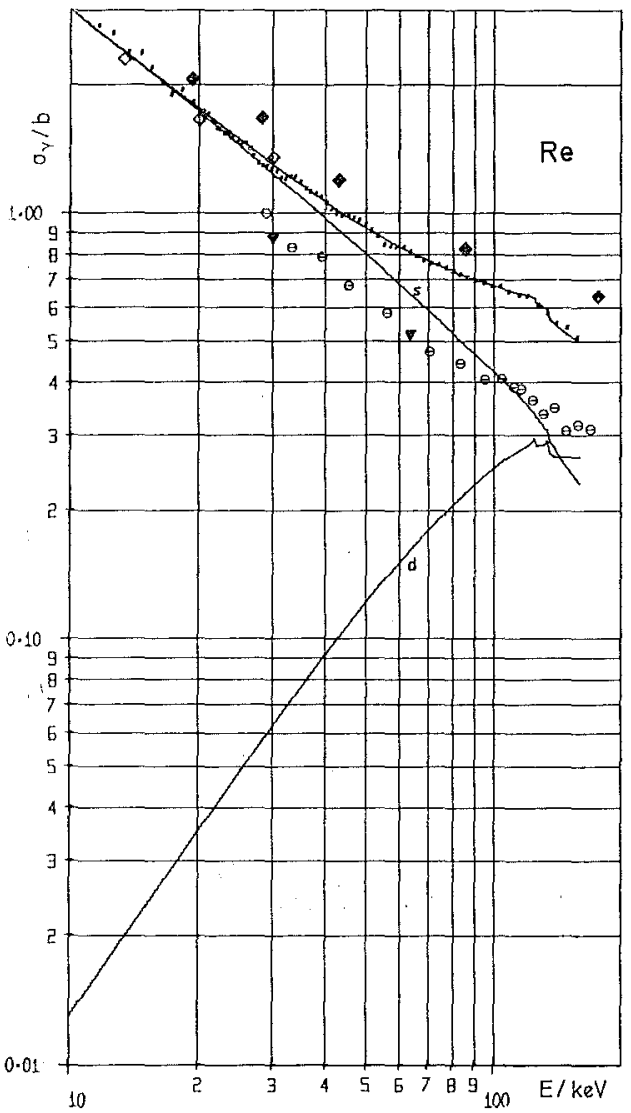

Fig. 17. Capture cross section of Re: $\$$ Macklin et al. $\left.{ }^{30}\right)$, Stupegia et al. ${ }^{33}$ ), $\diamond$ Friesenhahn et al. ${ }^{32}$ ), $\ominus$ Kononov et al. ${ }^{31}$ ).

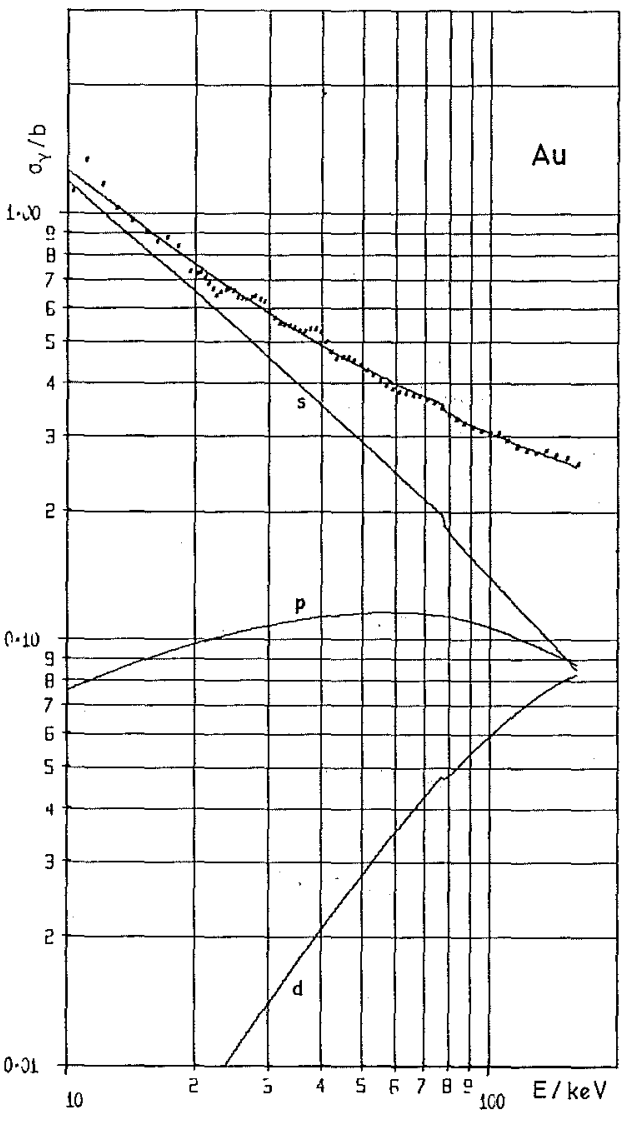

Fig. 18. Capture cross section of Au.

where $\Gamma_{\mathrm{n}^{\prime}}^{J \prime^{\prime} j^{\prime}}$ is the width for the inelastically scattered neutron $\mathrm{n}^{\prime}$ with channel spin $j^{\prime}$ and angular momentum $l^{\prime}$. The width fluctuation factor $F$ equals

$$
F=\left\langle\frac{\sum_{j} \Gamma_{\mathrm{n}}^{J l j} \Gamma_{\gamma}^{J}}{\Gamma^{J}}\right\rangle \mid \frac{\left\langle\sum_{j} \Gamma_{\mathrm{n}}^{J l}\right\rangle\left\langle\Gamma_{\gamma}^{J}\right\rangle}{\left\langle\Gamma^{J}\right\rangle} .
$$

Under some simplifying assumptions we will bring the expression (7) into a form 
suited for our purposes. We introduce the neutron width $\left\langle\Gamma_{\mathbf{n}}^{J l}\right\rangle$ averaged over the $\left(\varepsilon_{J}^{I l}=0,1,2\right)$ possible $j$-values for given $I, l, J$ :

$$
\left\langle\sum_{j} \Gamma_{\mathrm{n}}^{J l j}\right\rangle=\varepsilon_{J}^{I l}\left\langle\Gamma_{\mathrm{n}}^{J l}\right\rangle
$$

A $J$-dependence of $\left\langle\Gamma_{\mathrm{n}}^{J l}\right\rangle / D^{J}$ could not be verified definitely and is up to now in question. We shall make the usual assumption that it does not exist and introduce a $J$ independent neutron strength function $S_{l}$

$$
\frac{\left\langle\Gamma_{\mathrm{n}}^{J l}\right\rangle}{D^{J}}=\frac{\left\langle\Gamma_{\mathrm{n}}^{l}\right\rangle}{D}=S_{i} v_{l} \sqrt{ } E .
$$

The $v_{l}$ are the penetration factors (calculated for a nuclear radius $R=1.45 A^{\frac{1}{3}} \mathrm{fm}$ ).

The $\left\langle\Gamma_{\gamma}^{J}\right)$ can be replaced in good approximation by a non-fluctuating and $J$ independent $\Gamma_{\gamma}$.

The level density is assumed to be energy-independent over our limited energy range. For the $J$-dependence $f(J)$ of the level density $1 / D^{J}=f(J) / D$ the Fermi gas model gives

$$
f(J)=(2 J+1) \mathrm{e}^{-J(J+1) /\left(2 \sigma^{2}\right)},
$$

where $\sigma$ is the spin cut-off factor. Only for $J \ll \sigma$ we get the familiar approximation

$$
f(J) \approx 2 J+1 \text {. }
$$

Introducing the $\gamma$-strength function $S_{\gamma}=\Gamma_{\gamma} / D$ we may write for (7)

$$
\left\langle\sigma_{\gamma}^{J l}\right\rangle=2 \pi^{2} \hat{\lambda}^{2} g_{J} \frac{\varepsilon_{J}^{I l} S_{l} v_{l} \sqrt{E} S_{\gamma} f(J)}{\sum_{l^{*}} \varepsilon_{J}^{l^{*}} S_{l^{*}} v_{l^{*}} \sqrt{E}+\sum_{l^{\prime}} \varepsilon_{J}^{l^{\prime}} S_{l^{\prime}} v_{l^{\prime}} \sqrt{E^{\prime}+S_{\gamma} f(J)}} F .
$$

The total capture cross section is then

$$
\left\langle\sigma_{\gamma}\right\rangle=\sum_{J, l}\left\langle\sigma_{\gamma}^{J l}\right\rangle
$$

For a mixture of isotopes we have to average eq. (11) according to their abundances and make the somewhat questionable assumption that the $S_{l}, S_{\gamma}$ are equal for different isotopes. The first sum in the denominator of eq. (10) represents the elastic exit channels, the second one the inelastic exit channels. The summations are performed over those $l^{*}, l^{\prime}$ which are compatible with parity conservation. In the energy region investigated here only angular momenta $l, l^{*}, l^{\prime}$ up to 2 are important; higher partial waves can be neglected. Then we have for elastic scattering the selection rule

$$
\left|l-l^{*}\right|= \begin{cases}0 & \text { for } I=0 \\ 0,2 & \text { for } I \neq 0\end{cases}
$$


Since the penetration factors $v_{l}$ decrease rapidly with increasing $l$, the smallest $l^{*}$ will give the greatest contribution to the sum. For an incoming $d$-wave for example an elastically scattered out-going s-wave will predominate (for $I \neq 0$ ).

Thus up to three widths can occur in eq. (10) merely for elastic scattering and even more above an inelastic threshold. The great number of widths complicates the computation of the width fluctuation factor $F$ which is done by averaging over the $\chi^{2}$ distribution functions of degree $\varepsilon_{J}^{I I}$ for the neutron widths. The two-width case can be easily evaluated ${ }^{35}$ ). For the error and Placzek functions occurring in those expressions approximations by polynomials, rational functions and asymptotic expansions which are suited for computer calculations can be found in ${ }^{36}$ ). In all cases with more than two widths we have carried out the averaging for the calculation of $F$ by numerical integration.

\subsection{DETERMINATION OF STRENGTH FUNCTIONS}

Eqs. (10) and (11) were used to determine $S_{l}$ and $S_{\gamma}$ by a least-squares fit computer program. For a better understanding of the results of such calculations we will first discuss the energy dependence of $\left\langle\sigma_{\gamma}^{J l}\right\rangle$, neglecting inelastic scattering and the correction factor $F$. For sufficiently small energies $E$, where the sum over $l^{*}$ is much smaller than $S_{\gamma} f(J)$ and where $v_{l} \sim E^{l}$ :

$$
\left\langle\sigma_{\gamma}^{J l}\right\rangle \sim g_{J} S_{l} E^{l-\frac{1}{2}}
$$

At sufficiently large energies $S_{y} f(J)$ in the denominator of eq. (10) can be neglected. This can occur in our energy range for $\mathrm{s}-$ and p-waves. We obtain for this case:

$$
\left\langle\sigma_{\gamma}^{J l}\right\rangle \sim g_{J} f(J) S_{\gamma} \frac{1}{E} .
$$

We see that $\left\langle\sigma_{\gamma}^{J l}\right\rangle \sim S_{l}$ only for low energies where $\left\langle\sigma_{\gamma}^{J l}\right\rangle$ varies according to eq. (12) as $E^{l-\frac{1}{2}}$. However, in the region of the $1 / E$ dependence according to eq. (13), $\left\langle\sigma_{\gamma}^{J l}\right\rangle$ depends merely on $S_{\gamma}$ and within the approximation of eq. (13) not on $S_{l}$. This occurs for s-waves in the $\mathrm{keV}$ region so that a determination of $S_{0}$ from our measurements is impossible. For higher $l$-waves $\left\langle\sigma_{\gamma}^{J l}\right\rangle$ has a maximum between the regions of eq. (12) and eq. (13). This causes a bump in $\left\langle\sigma_{\gamma}\right\rangle$. The characteristic shape of the cross section allows a decomposition into partial waves and the determination of the strength functions $S_{l}$. The energy region near the maximum of the corresponding partial-wave cross section or just below is most suited for this purpose because of the large contribution of that partial wave to the total capture cross section. Therefore the energy region investigated here allows mainly a determination of the $\mathrm{p}$ - and d-wave strength functions $S_{1}$ and $S_{2}$.

The onset of inelastic scattering which causes a steeper decrease in the capture cross section may appear as a further complication for the analysis. However, it may increase the accuracy of the analysis since its influence on the various partial waves is different. 
For the fit according to eqs. (10) and (11) we need the spin cut-off parameter $\sigma$. Unfortunately reliable and complete information about $\sigma$ is not available for all nuclei. Usually the validity of eq. (9) is assumed ${ }^{8,37-39}$ ) in this context. However, for nuclei like $\mathrm{Nb}$ and In this assumption should not be valid because of their high spin $\left(I=\frac{9}{2}\right)$. We have assumed a constant value $\sigma=4$. In view of the data on $\sigma$ compiled in ${ }^{40}$ ) and their large errors this seems to be a good estimate for our atomic mass region. The uncertainty in $\sigma$ does not affect the $S_{l}$ strongly but it does affect $S_{\gamma}$. Since only the product $S_{\gamma} f(J)$ occurs, variations in the distribution $f(J)$ can be largely compensated by $S_{\gamma}$. Test calculations with $\sigma=4$ and $\sigma=\infty$ gave differences in the fitted $S_{\gamma}$ up to a factor 2.5 , whereas $S_{1}$ changed by not more than $12 \%, S_{2}$ even less. Therefore the values for $S_{\gamma}$ obtained by fitting an average capture cross section should be used with caution. For example Gibbons et al. ${ }^{8}$ ) have stated that their fitted values for $S_{\gamma}$ especially in the rare-earth region disagree sometimes by a factor of 4 with the results from other experiments; this fact might be related to the above problem.

TABLE 2

Results of the analysis of average capture cross sections

\begin{tabular}{|c|c|c|c|c|c|}
\hline \multirow[t]{2}{*}{ Element } & \multirow[t]{2}{*}{$A$} & \multirow{2}{*}{$\begin{array}{c}S_{0} \cdot 10^{4} \\
\left.\left(\text { from ref. }{ }^{40}\right)\right)\end{array}$} & \multicolumn{3}{|c|}{ Fitted parameters } \\
\hline & & & $S_{1} \cdot 10^{4}$ & $S_{2} \cdot 10^{4}$ & $S_{\gamma} \cdot 10^{4}$ \\
\hline $\mathrm{Nb}$ & 93 & 0.4 & $11.0 \pm 3.2$ & $0.4 \pm 0.3$ & $2.2 \pm 0.3$ \\
\hline Mo & 95.9 & 0.6 & $7.4 \pm 2.1$ & $0.43 \pm 0.13$ & $1.3 \pm 0.2$ \\
\hline $\mathrm{Pd}$ & 106.4 & 0.35 & $5.8 \pm 0.8$ & $1.3 \pm 0.3$ & $4.6 \pm 0.6$ \\
\hline Ag & 107.9 & 0.45 & $7.5 \pm 1.3$ & $3.1 \pm 1.3$ & $15.0 \pm 2.2$ \\
\hline $\mathrm{Cd}$ & 112.4 & 0.4 & $5.4 \pm 1.2$ & $1.4 \pm 0.5$ & $3.3 \pm 0.4$ \\
\hline In & 114.8 & 0.5 & $3.8 \pm 0.6$ & $0.93 \pm 0.6$ & $10.4 \pm 1.3$ \\
\hline Cs & 133 & 0.7 & $3.9 \pm 1.0$ & $2.4 \pm 1.5$ & $6.5 \pm 1.0$ \\
\hline Hf & 178.6 & 2.5 & $0.13 \pm 0.06$ & $2.1 \pm 0.7$ & $24 \pm 3$ \\
\hline $\mathrm{Ta}$ & 181 & 2.1 & $0.1 \pm 0.04$ & $2.2 \pm 0.5$ & $24 \pm 3$ \\
\hline W & 183.9 & $2: 1$ & $0.32 \pm 0.09$ & $0.7 \pm 0.15$ & $8.3 \pm 1.2$ \\
\hline $\operatorname{Re}$ & 186.2 & 2.4 & $<0.1$ & $6.3 \pm 1.0$ & $43 \pm 6$ \\
\hline $\mathrm{Au}$ & 197 & 1.6 & $0.19 \pm 0.04$ & $1.4 \pm 0.4$ & $15.6 \pm 2.0$ \\
\hline
\end{tabular}

For the least-squares fit, our experimental data were weighted with their error. The s-wave strength function $S_{0}$ which is an input parameter here is taken from a compilation by Seth ${ }^{41}$ ). The fitted parameters $S_{\gamma}, S_{1}, S_{2}$ and their calculated errors are shown in table 2 ; the best-fit cross-section curves and their three partial wave components are drawn as full lines in figures 7-18.

Inelastic scattering was taken into account for $\mathrm{Nb}, \mathrm{Ag}, \mathrm{Cs}, \mathrm{Hf}, \mathrm{Ta}, \mathrm{W}, \mathrm{Re}$ and $\mathrm{Au}$. The parameters for the corresponding energy levels were taken from ${ }^{48}$ ). Sometimes a sharp drop at the inelastic threshold is observed, especially in cases where elastically outgoing higher partial waves are competing with inelastically scattered s-waves. This effect is diminished in cases of many isotopes. It is necessary to take into account inelastic scattering even in those cases where no sharp decrease in the cross section is 
observed, as for e.g. $\mathrm{Ag}, \mathrm{Cs}$ and $\mathrm{Au}$. A tentative neglect of inelastic scattering drastically changed our results for the strength functions in some cases.

Good agreement between fitted and experimental curves is obtained in most cases. Only for $\mathrm{W}$ and $\mathrm{Hf}$ is the agreement not good above the inelastic threshold. However, these are elements with many isotopes, so that the assumption of equal average parameters for all isotopes might not be justified here.

In the higher-energy region the $\mathrm{d}$-wave contribution to the capture cross section is comparable to those of the other partial waves. Therefore a neglect of d-waves is not justified and this would deteriorate also the results for the p-wave strength functions $S_{1}$, since part of the d-wave contribution might then be incorrectly assigned to the $\mathrm{p}$-wave. The errors in the determined d-wave strength functions $S_{2}$ are large, especially in those cases where the d-wave contribution is small. An extension of the experiment to higher energies could improve this accuracy.

The energy region is also not optimal for the determination of the p-wave strength function $S_{1}$ in some cases, especially for large $S_{1}$. At $10 \mathrm{keV}$, the p-waves for $\mathrm{Nb}$ and Mo, for instance, have already exceeded the energy region of the maximum. Their contributions are then only weakly dependent on $S_{1}$. Moreover, the structure in the cross sections unfortunately becomes more pronounced at these low energies and may not be sufficiently eliminated by averaging over a large enough region. This results in a rather large error in $S_{1}$. Since this argument also applies to other measurements in the same energy region ${ }^{8}$ ), it might explain some of the discrepancies for $S_{1}$ near its maximum around mass $A=95$. An extension of the experiment in its present form to lower energies which would improve the accuracy in $S_{1}$ is difficult with the Van de Graaff accelerator.

The p-wave strength functions $S_{1}$ from this work are compared with some results of other groups and a calculated curve in fig. 19. Gibbons et al. ${ }^{8}$ ), Weston et al. ${ }^{38}$ ), and Popov et al. ${ }^{39}$ ) analysed capture cross sections, Seth et al. ${ }^{42}$ ) used total cross sections. All authors found the maximum near $A=95$, predicted by the optical model, but with appreciable differences in peak height and form. There are large uncertainties, and the experimental points are widely scattered. But there are also systematic deviations between the data frcm $\sigma_{\gamma}$ and $\sigma_{\mathrm{t}}$ measurements: All $\sigma_{\gamma}$ experiments indicate a higher peak than those for $\sigma_{t}$. This difference cannot be explained in all cases by an unfavourable energy range and corresponding large errors. Our results agree fairly well with the results of the Russian group ${ }^{39}$ ) for $\mathrm{Nb}, \mathrm{Ag}$, In, and Cs which were obtained from lead spectrometer measurements down to low energies. Only their results for ${ }^{98} \mathrm{Mo}$ and ${ }^{100} \mathrm{Mo}$ are far below those of the present work and those of all other authors.

The theoretical curve was calculated by Perey and Buck ${ }^{43}$ ) from a nonlocal optical potential. Its peak height near $A \approx 95$ is lower than our values and those of most other authors; in the region of the minimum around $A \approx 180$ it is larger than all experimental results.

Only few results for d-wave strength functions have been reported; they are compared with our data in fig. 20 . The $\mathrm{d}$-wave contribution has often not been taken into 
account in the analysis of capture cross sections even up to energies above the range investigated here. That this is unjustified is indicated by deviations between experimental and fitted curves at higher energies. Seth et al. ${ }^{42}$ ) have determined d-wave strength functions for various elements from an analysis of total cross-section measurements. Their results for $S_{2}$ show, similar to those for $S_{1}$, a trend to lower values than ours. Kononov ${ }^{44}$ ) has obtained $S_{2}$ values by an analysis of capture cross sections taking into account inelastic scattering, as was done in the present work.

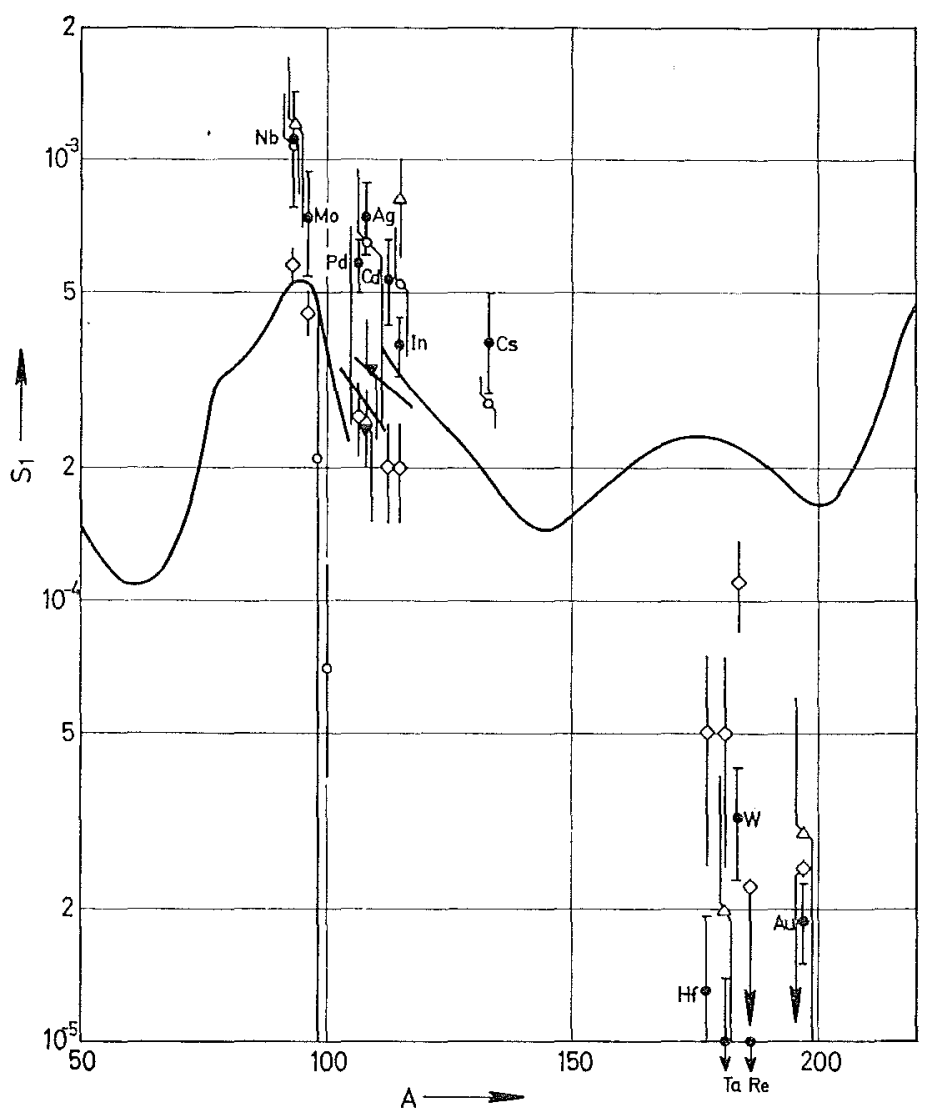

Fig. 19. p-wave strength functions from $\sigma_{y}:$ this work, $\triangle$ Gibbons et al. $\left.{ }^{8}\right), \nabla$ Weston et al. ${ }^{37}$ ), $\circ$ Popov et al. ${ }^{39}$ ), from $\sigma_{\mathrm{t}}: \diamond$ Seth et al. ${ }^{42}$ ), - calculation of Buck and Perey ${ }^{43}$ ).

The theoretical curve for $S_{2}$ was drawn with the transmission coefficients that Auerbach and Perey have calculated for a non-local optical potential ${ }^{45}$ ). The agreement with the experimental results is not very good. Values from some experiments are appreciably below the theoretical curve. There is a similar trend of experimental results for $S_{0}$ and $S_{1}$ towards values below the theoretical minima. Similar to the p-wave case some of our results are again above the calculated strength function curve 
and the results from $\sigma_{t}$ measurements. However, the few scattered experimental points for $S_{2}$ with large uncertainties are not sufficient for a general conclusion.

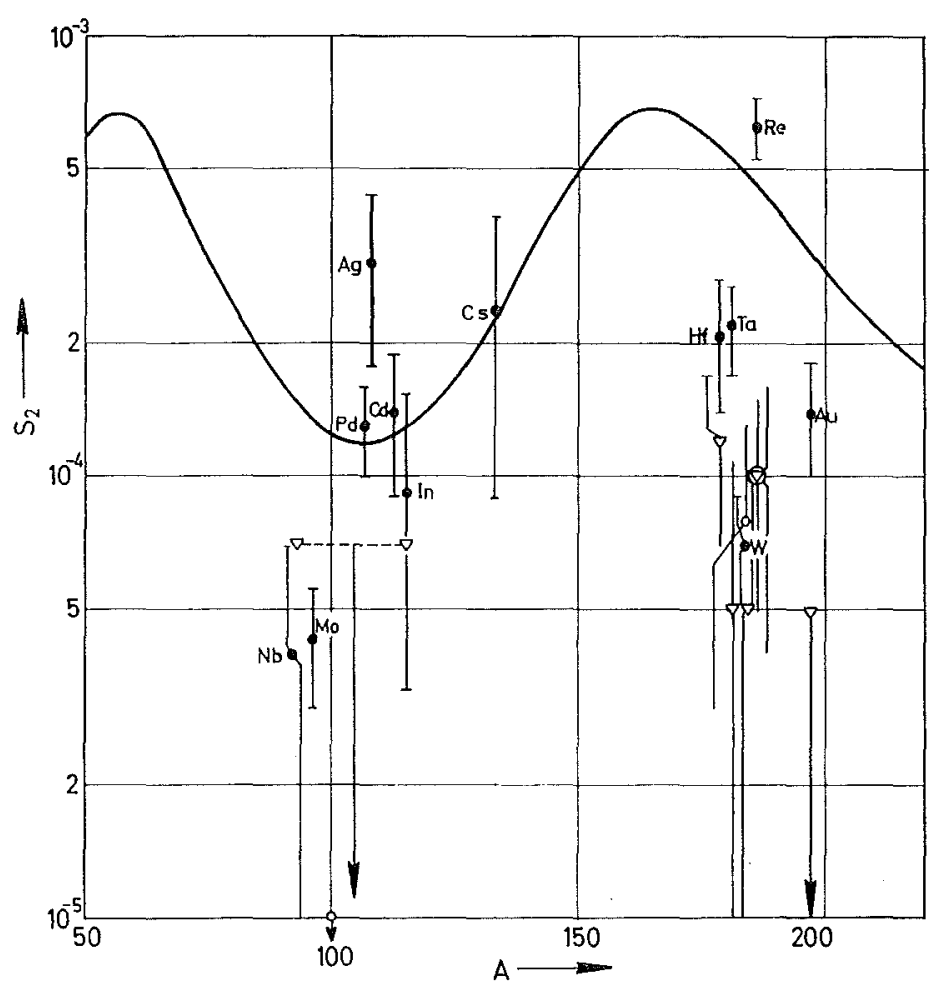

Fig. 20. d-wave strength functions: - this work, $\diamond$ Seth et al. ${ }^{42}$ ), O Kononov ${ }^{44}$ ), - calculated from Auerbach et al. ${ }^{45}$ ).

It should be realized that deviations from smooth strength function curves as calculated from the optical model are not necessarily due to experimental error but may be real, since the optical model does not describe all details of nuclear structure, but only the gross features. For example investigations at the maximum of the s-wave strength function around $A=55$ indicate that large fluctuations in the theoretical curve for $S_{0}$ may arise when shell model effects are taken into account ${ }^{46}$ ).

The author wishes to thank Prof. Dr. Beckurts for his encouragement and many suggestions on this work.

\section{References}

1) H. W. Schmidt and C. W. Cook, Nucl. Phys. 20 (1960) 202

2) T. C. Belanova, A. A. Banjkov, F. F. Michailus and J. J. Stavisskii, Conf. on Nucl, data for reactors, $\mathrm{CN}-23 / 96$ (1966)

3) D. Bogart, Conf. on Nucl. data for reactors, CN-23/96 (1966) 
4) F. H. Fröhner, GA-8072 (1967)

5) A. I. Isakov, Yu. P. Popov and F. L. Shapiro, ZhETF (USSR) 38 (1960) 989

6) F. Mitzel and H. S. Plend1, Internal report KFK/INR $81 / 64$

7) B. C. Diven, Int. Conf. on the study of nuclear structure with neutrons, Antwerp (1965)

8) J. H. Gibbons, R. L. Macklin, P. D. Miller and J. H. Neiler, Phys. Rev. 122 (1961) 182

9) R. C. Block, G. G. Slaughter, L. W. Weston and F. C. Vonderlage, Proc. Symp. on neutr time-of-flight methods, ed. by Spaepen (1961) 203

10) E. Haddad, R. B. Walton, S. J. Friesenhahn and W. M. Lopez, Nucl. Instr. 31 (1964) 125

11) M. C. Moxon and E. R. Rae, Nucl. Instr. 24 (1963) 445

12) H. Weigmann, G. Carraro and K. H. Böckhoff, Nucl. Instr. 50 (1967) 265

13) R. L. Macklin and J, H. Gibbons, Phys. Rev. 159 (1967) 1007

14) H. Faissner, F. Ferrero, A. Ghani and M. Reinharz, Nucleonics 21 (1963)

15) G. Rohr, Internal report KFK/IAK $47 / 67$

16) R. L. Macklin, Nucl. Instr. 26 (1964) 213

17) L. Dresner, Nucl. Instr. 16 (1962) 176

18) H. W. Schmidt, ORNL-2883 (1960)

19) H. O. Zetterström, S. Schwartz and L. G. Strömberg, Nucl. Instr. 42 (1966) 277

20) W. P. Poenitz, Conf. on Nucl. data for reactors, CN-23/6 (1966)

21) W. P. Poenitz, D. Kompe and H. O. Menlove, J. Nucl. Energy 22 (1968) 505

22) W. P. Poenitz, Nucl. Instr. 58 (1968) 39

23) J. Spaepen, Conf. on Nucl. data for reactors, CN-23/119 (1966)

24) S. A. Cox and F. R. Pontet, J. Nucl. Energy A/B21 (1967) 271

25) A. Bergström, S. Schwartz and L. G. Strömberg, CCDN-NW 3 (1966)

26) D. Kompe, Conf. on Nucl. data for reactors, CN-23/10 (1966)

27) P. H. White, J. Nucl. Energy A/B 19 (1965) 325

28) Yu. P. Popov and F. L. Shapiro, JETP (Sov. Phys.) 15 (1962) 683

29) R. Booth, W. P. Ball and M. H. MacGregor, Phys. Rev. 112 (1958) 226

30) R. L. Macklin, J. H. Gibbons and T. Inada, Phys. Rev. 129 (1963) 2695

31) V. N. Kononov, Yu. Ya. Stavisskii, S. R. Chistozvonov and V. S. Shorin, Conf. on Nucl. data for reactors, CN-23/99 (1966)

32) S. J. Friesenhahn, D. A. Gibbs, E. Haddad, F. H. Fröhner and W. M. Lopez, J. Nucl. Energy 22 (1968) 191

33) D. C. Stupegia, M. Schmidt and A. A. Madson, J. Nucl. Energy A/B 19 (1965) 767

34) A. M. Lane and J. E. Lynn, Proc. Phys. Soc. 70A (1957) 557

35) L. Dresner, ORNL-2659 (1959)

36) M. Abramowitz and I. A. Stegun, Handbook of mathematical functions, AMS 55, Nat. Bur. of Stand.

37) E. Bilpuch, L. W. Weston and H. W. Newson, Ann. of Phys. 10 (1960) 455

38) L. W. Weston, K. K. Seth, E. G. Bilpuch and H. W. Newson, Ann. of Phys. 10 (1960) 477

39) Yu. P. Popov and Yu. I. Fenin, JETP (Sov. Phys.) 16 (1963) 1409

40) M. Bormann and H. Neuert, Fortschr. der Phys. 11/7 (1963) 277

41) K. K. Seth, Nucl. Data A2/3 (1966)

42) K. K. Seth, R. H. Tabony, E. G. Bilpuch and H. W. Newson, Phys. Lett. 13 (1964) 70

43) B. Buck and F. Perey, Phys. Rev. Lett. 8 (1962) 444

44) V. N. Kononov, Sov. J. of Nucl. Phys. 5 (1967) 89

45) E. H. Auerbach and F. G. J. Perey, BNL 765 (T-286) (1962)

46) K.-N. Müller, private communication

47) H. Weigmann and H. Schmidt, Nucl. Phys. A104 (1967) 513

48) C. M. Lederer, J. M. Hollander and I. PerIman, Table of Isotopes, 6th ed., (1967)

49) G. W. Grodstein, NBS 583 (1957) 